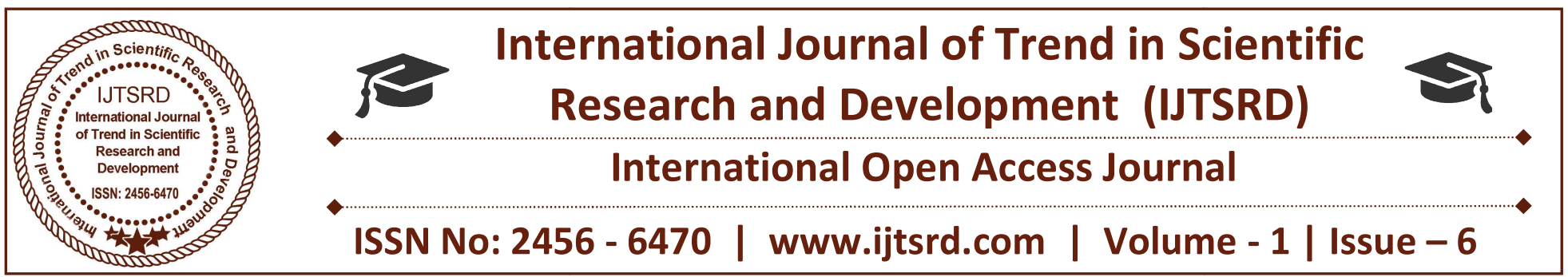

\title{
A Research on Cluster Based Routing Methodologies for Wireless Sensor Networks
}

Venkateswarulu Naik. B

Assistant Professor, C.S.E Dept, Mallareddy Institute of Technology And Sciences, Secunderabad, India

\author{
G. Ravi Kumar \\ Assistant Professor, C.S.E Dept, Mallareddy Institute \\ of Technology And Sciences, Secunderabad, India
}

\begin{abstract}
The design of Wireless sensor networks has currently significant attention due to their limitless potential and has great value of applications such as not closely connected environmental monitoring and target tracking. This has been motivated by the development and availability of small, low cost and intelligent sensors. A wireless sensor network is a collection of sensor nodes prearranged into a cooperative network. These sensors nodes are outfitted with limited processing and computing resources. Since radio transmission and reception consumes a lot of power, one of the critical suspicions in wireless sensor network is the inherent limited battery power within the sensor nodes. Therefore battery power is one of the vital parameter for an algorithm design to increase the lifespan of the sensor node. Recent researches have been carried out in investigating diverse aspects in connection with low power routing protocols. In this paper, the focus is mainly driven towards the review of energy efficient cluster-based available routing protocols for wireless sensor network. Also a methodology by which optimal routing can be achieved in the context of energy is portrayed artistically.
\end{abstract}

Keywords: Wireless sensor network, network life span, cluster-based routing, distributed clustering, energy efficiency

\section{INTRODUCTION}

In recent years Wireless sensor networks have gained world-wide range of attention, particularly with the proliferation in Micro-Electro-Mechanical Systems
(MEMS) technology, which has facilitated the development of smart sensors. A WSN is a set of hundreds or thousands of sensor nodes working together to monitor a region to obtain data about the environment. These sensor nodes have capabilities of sensing, establishing wireless communication between each other and doing computational and processing operations [1]. The potential applications of WSNs are exceedingly varied, such as ecological monitoring, traffic monitoring (figure 1), tracking and military surveillance [2]. When placed in unpleasant physical environment it becomes impossible to charge or replace the sensor nodes. Therefore it is desirable to design a communication network protocol such that energy source is used efficiently to maximize the lifetime of the network. Another crucial issue is the data delivery time by sensor nodes, especially in battle field, medical applications and border security applications, where minimum delay is desirable [3]. Sensors in such networks are equipped with sensing, information processing and radio broadcast while the power is highly inadequate. Due to the sensors ${ }^{\text {ce }}$ limited power, advanced system that improves energy competence to extend the network lifetime is highly mandatory. Thus energy-awake policy has been a hot research area at all layers of the networking protocol stack. Sensor nodes are usually scattered in a sensor field and each sensor node comprises of sensing, processing, transmission, mobilize, position finding system and power units. Sensor nodes coordinate among themselves to produce high-quality information about the physical environment [4]. Each sensor node bases its decisions on the assignment, information it presently has with its acquaintance of 
sensing, computing, communication and energy sources. Each of these dispersed sensor nodes has the ability to collect and route data either to other sensors or to the base station (BS). A BS may be a stable node capable of connecting the sensor network to a current communication organization [4].

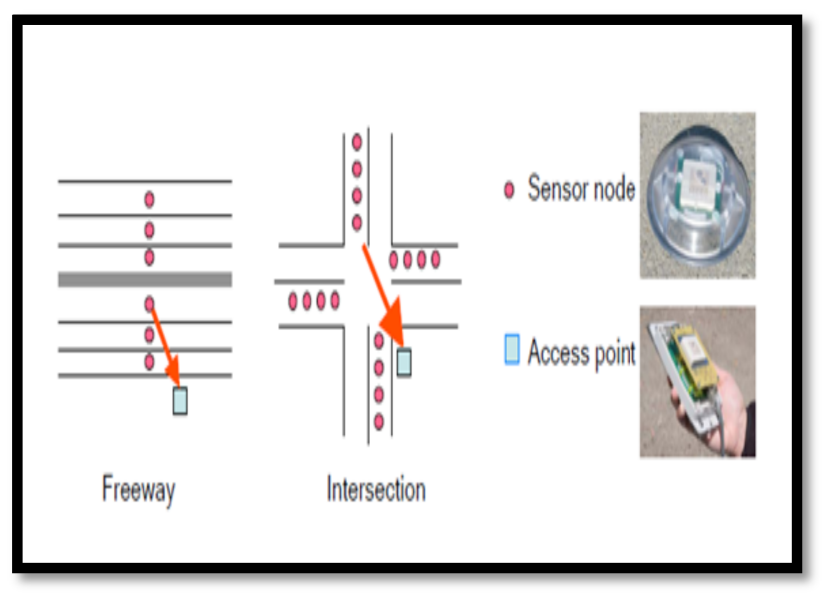

\section{Figure 1: Application of WSN in traffic measurement}

However, sensor nodes are constrained in energy supply and bandwidth. Thus, a modern technique that excludes energy inadequacy and shortened lifetime of the network is highly required. In the network layer, it is highly desirable to find methods for energyefficient route discovery and relaying of data from sensor nodes to the BS. This paper aims to introduce two newly evaluative indexes related to network coverage rate and effective network lifetime together with a better characterization of the pros and cons of a routing protocol. Meanwhile, this paper presents a new protocol: Clustering Patch Hierarchical Routing Protocol (CPHRP) and uses newly evaluative standard to demonstrate the superiority of its performance. The remainder of this paper is organized as follows. Section I describes the design of communication network protocol such that energy source is used efficiently to maximize the lifetime of the network. Section II describes the outline on the classification of routing protocols design and their challenges. Section III portrays a study of an energy efficient clustering structure and its comparison with two well evaluated clustering and routing protocols LEECH and HEED. Section IV describes the problem in currently evaluative standard and the realization of CPHRP with few properties of new CPHRP. Section V concludes the paper with a quantity of directions for future work

\section{CLASSIFICATION OF ROUTING PROTOCOLS}

There are many routing protocols for WSN mainly for static networks and there are little protocols for networks with dynamicity. All major protocols [5] may be categorized into four categories.

\section{A. Data Centric Protocols}

Data is usually transmitted from every sensor node within the distribution region with repetition. Since this is very ineffective in terms of energy utilization, routing protocols will be able to select a set of sensor nodes and utilize data aggregation during relaying of the data. This consideration has led to data-centric routing, which is not the same from conventional address-based routing where routes are created between addressable nodes managed in the network layer of the transmission heap. In data-centric routing, the sink sends queries to certain regions and waits for data from the sensors located in those particular regions [40]. Since data is being demanded through queries, characteristic based identification is needed to state the properties of data [7]. Representative protocols for data centric approach (Flooding and gossiping, SPIN, directed diffusion, rumor routing and energy aware routing).

\section{B. Hierarchal Protocols}

A single-tier network can cause the gateway to overload with the increase in sensors ${ }^{\text {ee }}$ mass. Such excess load might cause intermission in communication and poor tracking of events. In addition, a single-gateway architecture is not scalable for a bigger set of sensors covering a wider area of interest, since the sensors are typically not capable of long haul transmission. To allow the system to handle with added load and to cover a large area without degrading the service, clustering has been practiced in some routing methods. The foremost intention of hierarchical routing is to cost effectively maintain the energy consumption of sensor nodes by connecting them in multi-hop communication within a particular cluster. Also this is attained by performing data aggregation, reducing the number of data to be communicated to the sink or BS. Cluster formation is naturally based on the energy backup of the sensors and their vicinity to the $\mathrm{CH}[7,9]$. Few representative protocols for hierarchal based routing approach (LEACH, PEGASIS, HPEGASIS, TEEN and APTEEN). C. Location Based Protocols Most of the routing protocols for sensor networks require location information of the sensor nodes. In most cases locality 
information is needed in order to work out the distance between two particular nodes, so that energy consumption can be assessed. Since there is no addressing structure for sensor networks like IPaddress, location information can be applied in routing of data in an energy effectual manner. For instance, if the region to be sensed is known, using the location of sensors, the queries can be broadcasted only to that particular region which will exclude the number of transmission substantially [39]. Representative protocols for location based routing approach (MECN, SMECN, GAF and GEAR). D. Network flow and Quos Aware Protocols Although most of the routing protocols proposed for sensor networks fit the necessary classification, some pursue little different approach such as network flow and Quos [7]. In Copyright to IJIREEICE www.ijireeice.com 402 some approaches, route setup is modeled and solved as a network flow problem. Quos aware protocols consider end-to end delay requirements while setting up the paths in the sensor network. Representative protocols for network flow and Quos aware approach (Maximum life time energy routing protocol, maximum lifetime data gathering protocol, minimum cost forwarding, SAR and SPEED). 2.1 Routing Challenges and Design Issues in WSNs The design of routing protocols for WSN is inclined by many challenging factors. In order to achieve efficient communication, these factors must be considered to an extent. Some of the routing challenges and design issues that have an effect on routing process in WSNs [7] is elaborated as follows. 1) Node deployment in WSN is application dependent and affects the performance of the routing protocol. 2) Sensor nodes can use up their limited supply of energy to perform computations and transmitting information in a wireless environment. 3) Some sensor nodes may fail or be blocked due to lack of power, physical damage or environmental interference. 4) Since sensor nodes may create significant unwanted data, similar packets from multiple nodes can be aggregated to reduce the number of transmissions. 5) As the energy gets depleted, the network may be in need to reduce the quality of the output in order to reduce the energy dissipation of the nodes and hence lengthens the overall network lifetime. 6) A given sensor's view of the environment is limited both in range and accuracy, that it can only cover a limited physical environmental [15].

\section{Collocation Based Protocols}

Most of the routing protocols for sensor networks require location information of the sensor nodes. In most cases locality information is needed in order to work out the distance between two particular nodes, so that energy consumption can be assessed. Since there is no addressing structure for sensor networks like IP-address, location information can be applied in routing of data in an energy effectual manner. For instance, if the region to be sensed is known, using the location of sensors, the queries can be broadcasted only to that particular region which will exclude the number of transmission substantially [39].Representative protocols for location based routing approach (MECN, SMECN, GAF and GEAR).

\section{Network flow and Quos Aware Protocols}

Although most of the routing protocols proposed for sensor networks fit the necessary classification, some pursue little different approach such as network flow and Quos [7] 2.1 Routing Challenges and Design Issues in WSNs The design of routing protocols for WSN is inclined by many challenging factors. In order to achieve efficient communication, these factors must be considered to an extent. Some of the routing challenges and design issues that have an effect on routing process in WSNs is elaborated as follows.

1) Node deployment in WSN is application dependent and affects the performance of the routing protocol.

2) Sensor nodes can use up their limited supply of energy to perform computations and transmitting information in a wireless environment.

3) Some sensor nodes may fail or be blocked due to lack of power, physical damage or environmental interference.

4) Since sensor nodes may create significant unwanted data, similar packets from multiple nodes can be aggregated to reduce the number of transmissions.

5) As the energy gets depleted, the network may be in need to reduce the quality of the output in order to reduce the energy dissipation of the nodes and hence lengthens the overall network lifetime.

6) A given sensor's view of the environment is limited both in range and accuracy, that it can only cover a limited physical environmental [15]. 


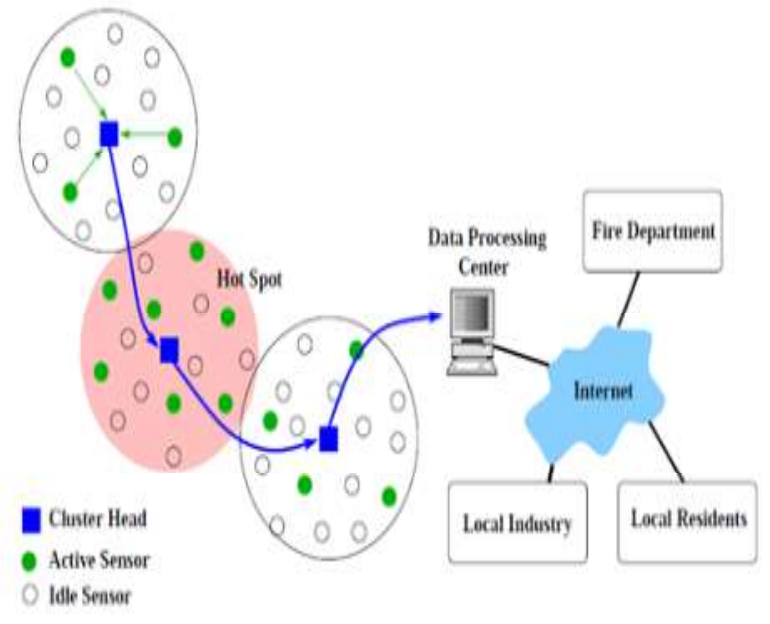

III. RELATED WORK

\subsection{Energy-Efficient Clustering Structures}

In this Paper, the focus is mainly driven towards the survey of energy efficient cluster based available routing protocols [6] for wireless sensor network by which optimal routing can be achieved in the context of energy. Traditional (or flat) routing protocols for WSN may not be optimal in terms of energy consumption. Clustering mechanism can be used as an energy-efficient communication protocol [8]. The focal objectives of clustering: to minimize the total transmission power of the nodes in the selected path and to balance the load among the nodes for prolonging the network lifetime. Clustering is a sample of layered protocols in which a network is composed of several clumps of sensors [11]. As depicted in figure 3 , each cluster is managed by a special node or leader, called cluster head, which is responsible for coordinating the data transmission activities of all the sensors in its cluster. All sensors in a cluster communicate with a $\mathrm{CH}$ that acts as a local coordinator or sink for performing intra-transmission arrangement and data aggregation Clustering mechanism of sensor nodes Cluster heads transmits the sensed data to the universal sink. The communication distance over which the sensors send their data to their $\mathrm{CH}$ is smaller compared to their particular distances to the universal sink [17]. Since a network is characterized by its limited wireless channel bandwidth, it would be useful if the amount of data transmitted to the sink is minimized. To achieve this goal, a local collaboration between the sensors in a cluster is required in order to reduce the bandwidth demands. As pictured in figure 3, clustering usually localizes the routing setup within the cluster and therefore it reduces the routing overhead by each node and the topology maintenance overhead. Using clustering, the network appears smaller and more stable. The information generated from neighboring sensor nodes, is often redundant and correlated, thereby data aggregation by each $\mathrm{CH}$ conserves communication bandwidth as well. Moreover, the ability to use different power levels in inter-cluster and intra cluster communication reduces the interferences and collisions in the network resulting in a better throughput [11]. Clustering is a challenging task [15] and CHs often lose more energy compared to regular nodes. It is necessary to perform re clustering periodically in order to select energyabundant nodes to serve as $\mathrm{CHs}$.

\subsection{Hierarchical Based Cluster Models}

A hierarchical approach breaks the network into clustered layers [10]. Nodes are grouped into clusters with a $\mathrm{CH}$ that has the responsibility of routing the data from one $\mathrm{CH}$ to the other $\mathrm{CH}$ or to the $\mathrm{BS}$. Data travels hierarchically from a bottom clustered layer to top one, while it hops from one node to another, as it hops from one layer to another it covers larger distances thereby moving the data faster to the BS. Theoretically, the latency in such models is much lesser than in the multiple hop systems. Clustering provides essential optimization capabilities at the $\mathrm{CH}$ level. In the cluster based hierarchical system, data is first aggregated in the cluster and then sent to a top level $\mathrm{CH}$. Comparison of Clustering and Routing Protocols (LEACH and HEED) Within a particular cluster organization, cluster communication may be intra-cluster transmission (single hop or multi-hop) or inter-cluster transmission [39]. Low Energy Adaptive Clustering Hierarchy (LEACH) [12] was the first clustering protocol proposed for minimizing energy utilization. It forms clusters by using a distributed algorithm, each node has a certain probability of becoming a $\mathrm{CH}$ per round, and the task of being a $\mathrm{CH}$ is rotated between the nodes. A non- $\mathrm{CH}$ node determines its cluster by choosing the $\mathrm{CH}$ that can be reached with the least communication energy consumption. In the data transmission stage, each $\mathrm{CH}$ sends the aggregated packet to the BS by single hop. Hybrid Energy-Efficient Distributed (HEED) [13] clustering approach is one of the most recognized energy-efficient clustering protocols. It extends the basic scheme of LEACH by using left out energy and node compactness. In HEED [20] the initial probability for each node to become a provisional $\mathrm{CH}$ depends on its residual energy, and final $\mathrm{CHs}$ are 
selected according to the intra-cluster communication cost. The clustering procedure is divided into a number of iterations, and terminates within a fixed number of iterations. HEED accomplishes quite uniform distribution of $\mathrm{CHs}$ across the network. In both LEACH and HEED protocols (table 1), the basic difference is that while finding out the cluster node in a network, maximum residual energy at the node is used.

Table 1: Parameters comparison of LEACH and HEED

\begin{tabular}{|l|l|l|}
\hline Parameters & LEACH & HEED \\
\hline Scalability & Very Low & Moderate \\
\hline Delivery Delay & Very Small & Moderate \\
\hline Load Balancing & Moderate & Moderate \\
\hline $\begin{array}{l}\text { Algorithm } \\
\text { Complexity }\end{array}$ & Low & Moderate \\
\hline Parameters & LEACH & HEED \\
\hline $\begin{array}{l}\text { Energy } \\
\text { Efficiency }\end{array}$ & Very Low & Moderate \\
\hline Cluster Stability & Moderate & High \\
\hline
\end{tabular}

\section{PROBLEMS IN THE CURRENTLY EVALUATIVE STANDARD FOR SENSOR NETWORKS}

Hybrid Energy Efficient Distributed clustering (HEED) extends the basic scheme of LEACH by using residual energy and nodese degree or density as a performance metric for $\mathrm{CH}$ selection to achieve energy balancing. It operates in multiple hop networks, using a modifying transmission power in the inter-clustering communication. HEED was intended with four primary goals: (i) prolonging network lifetime by distributing energy consumption (ii) terminating the clustering process within a constant number of iterations (iii) minimizing control overhead and (iv) producing well distributed CHs. In HEED, the formulated algorithm periodically selects CHs according to a combination of two clustering parameters. The primary parameter is the residual energy of each sensor nodes (used in calculating probability of becoming a $\mathrm{CH}$ ) and the secondary parameter is the intra-cluster communication cost as a function of cluster density or node degree (i.e. the number of neighbors). The former is used to probabilistically select an initial set of $\mathrm{CH}$ shile the latter is used for breaking the ties. In HEED, the clustering process at each sensor node requires several rounds. Every round is quite long enough to receive messages from any neighbor within the cluster range As in $\mathrm{LEACH}$, an initial percentage of $\mathrm{CHs}$ in the network CThresh is predefined. The parameter CThresh is only used to limit the initial $\mathrm{CH}$ announcements and has no direct impact on the final cluster structure. In HEED Copyright to IJIREEICE www.ijireeice.com 404 [21], each sensor node sets the probability $\mathrm{CHThresh}$ of becoming a $\mathrm{CH}$ (equation 1) $x=($ ) / CH C E Thresh Thresh Residual max (1) where $\mathrm{E}$ Residual is the estimated current residual energy in the sensor node and $\mathrm{E}$ max is the maximum energy corresponding to a fully charged battery, which is same as that for homogeneous sensor nodes. The CHThresh value must be greater than a minimum threshold Pin. A $\mathrm{CH}$ is either an indefinite $\mathrm{CH}$ if its CHThresh $<1$, or a final $\mathrm{CH}$ if its CHThresh has reached unity. In the course of each round of HEED, every sensor node that never heard from a $\mathrm{CH}$, concludes itself to become a $\mathrm{CH}$ with probability $\mathrm{CHThresh}$. The newly selected $\mathrm{CHs}$ are added to the current set of CHs. If a sensor node is selected to become a $\mathrm{CH}$, it broadcasts a declaration message as an indefinite $\mathrm{CH}$ or a final $\mathrm{CH}$. A sensor node listening the $\mathrm{CH}$ list selects the $\mathrm{CH}$ with lowest cost from this set of CHs. Every node then doubles its CHThresh and goes to the next step of a node completes the execution without electing it to become a $\mathrm{CH}$ or joining a cluster, it announces itself as a final $\mathrm{CH}$. A node can be selected as a $\mathrm{CH}$ at each successive clustering interval, if it has higher residual energy with lower cost. However the $\mathrm{CH}$ selection accords with only a subclass of parameters, which can feasibly execute control on the system. These methods are suitable for extending the lifetime rather than for the entire needs of WSN [35].

\subsection{Inaccurate Evaluation for Self-Protocols}

The simulation results of HEED with 100, 200, and 400 nodes, shows the network lifetime is not extended by $7 \%$ with the growth of number of nodes. It is confirmed that the improvement in network performance is not with the growth of the number of nodes. However, the network load becomes more and more unbalanced with the increase of sensor nodes. The decline occurs in the performance of network load with the growth of the nodes, this will conflict with the conclusion. With multiple increasing of nodes, the network lifetime does not grow in a multiplied speed, even at very small increase. After these analyses, it is seen that as 
the number of nodes increases (figure 4) there is a significant growth in sensor-nodes and energy consumption. But this technique improves the network coverage rate and effective network lifetime with the increase of nodes and hence the rise in cost for network [37] happens. Therefore rise in the number of nodes and costs cannot give a satisfactory performance, thus the network-load of HEED [20] is unbalanced, it will be increasingly unbalanced with the growth of sensor nodes. The reason found to be behind this is the handicap of one-hop model. A cluster that is far away from the BS is always premature death [37]. Figure 4: Network life time of HEED

\subsection{Realization of CPHRP and its Analysis Clustering Patch}

Hierarchical Routing Protocol (CPHRP) [24] progress network coverage rate and effective network lifetime in WSNs through clustering patch, and it has hierarchical multi-path tree routing characteristics. It partitions the nodes into three classes: cluster node, sense node and non-sense node, which increases the energy conservation [34]. In CPHRP, a cluster can be covered by at least three inner-cluster sense nodes or up to six inner-cluster sense nodes. Therefore, letting the densely scattered nodes be in completely sensing condition. Figure 5 shows that inner-cluster nodes and $\mathrm{CH}$ within a distance $\mathrm{d} €(0, \mathrm{R})$, so $\alpha=2$ arc cos $(d / 2 R)$, namely $€(2 \pi / 3 \pi)$. When the distance of the inner-cluster nodes to each other's is $\mathrm{R}$, the distance from inner-cluster nodes to $\mathrm{CH}$ is $\mathrm{R}$ and the sensing radius is $\mathrm{R}$, then each cluster can be covered by up to six inner-cluster sense nodes constituting an equilateral hexagon. CHThresh is a constant 0.05, Emax is the initial energy. When there are some nodes being not covered by the cluster, nodes advertise itself as a $\mathrm{CH}$ to patch for outside the monitoring areas according to CHThresh. When the node does not belong to any cluster, not a cluster head, CHThresh1, then the node advertises itself as a cluster head directly, only the cluster nodes are let to become sense node and the non-cluster nodes become non-sense node [34]. 4.3 Simulation results for effective rounds and network life time (HEED and CPHRP) Figures 6 and 7 shows the comparison of the effective network lifetime between CPHRP and HEED in different scenarios required by the network coverage rate and finds significant improvement in CPHRP with wider range of applications. Meanwhile the successful network lifetime of CPHRP rises by more than $60 \%$ compared with peer network with the multiple growth of network nodes. However, HEED did not show much improvement in network lifetime and the progress of growth is much slower compared to the CPHRP protocol.

\section{CONCLUSION AND FUTURE RESEARCH}

Owing to scarce energy resources of the sensor nodes, energy efficiency is one of the main issues in the design of protocols for WSNs. The ultimate goal behind the protocol design is to keep the sensor nodes operating for as long as possible to extend the network lifetime. In this paper, a survey and summarization of recent research works is carried out, focused mainly on the energy efficient hierarchical cluster-based routing protocols for WSNs. The nodes are partitioned into three classes: cluster node, sense node and non-sense node, in order to improve energy conservation. The newly evaluative standard CPHRP, guarantees more than $90 \%$ network coverage rate with better network lifetime, when the number of innercluster sense nodes is 6 . With the multiple growth of network nodes, the efficient network lifetime of CPHRP rises by more than $60 \%$. When the number of inner-cluster nodes increases in multiples, the growth of its network lifecycle is more than $75 \%$ in contrast with HEED.

\section{REFERENCES}

1) Shim Kumar Singh, M.P.Singh, D.K.Singh, (2010), "A survey of energy efficient hierarchical cluster-based routing in wireless sensor network", International Journal of Advanced Networking and Applications, Volume 02, Number 02, Pages $570-580$.

2) Jennifer Yuck, Biswanath Mukherjee, Dipak Ghostly, (2008), "Wireless sensor network survey", Computer Networks, Department of Computer Science, University of California, Pages 2292-2330.

3) F.Bajaber, Ibadan, (2009), "Centralized dynamic clustering for wireless sensor networks", Proceedings of the International Conference on Advanced Information Networking and Applications.

4) F.Akyildiz, W.Su, Y.Sankarasubramaniam, E.Cayirci, (2004), "A survey on sensor network", IEEE Communication Magazine, Volume 40, Number 8, Pages 102-114. 
International Journal of Trend in Scientific Research and Development (IJTSRD) ISSN: 2456-6470

5) J.Al-Karaki, A.Kamal, (2004), "Routing techniques in wireless sensor networks: a survey", IEEE Communications Magazine, Volume 11, Number 6, Pages 6-28.

6) M.P.Singh, M.M.Gore, (2010), "A new energyefficient clustering protocol for wireless sensor networks", International Journal of Advanced Networking and Applications, Volume 02, Number 02, Pages 570-580.

7) E.Royer, Catch, (2009), "A review of current routing protocols for dhow mobile wireless networks", IEEE Personal Communications, Volume 6, Number 2, Pages 4655.

8) S.Bandopadhya, E.Coyle, (2003), "An energy efficient hierarchical clustering algorithm for wireless sensor networks", in Proceeding of IEEE INFOCOM, Volume 3, Pages 1713-1723.

9) A.Manjeshwar, D.P.Agarwal, (2001), "TEEN: a routing protocol for enhanced efficiency in wireless sensor networks", in 1st International Workshop on Parallel and Distributed Computing Issues in Wireless Networks and Mobile Computing.

10) Pawn Kumar Kocher, Simarpreet Kauri, NiranjanYadav, "Cluster based energy efficient protocol for wireless sensor network", International Journal of Research in Engineering and Applied Sciences.

11) W.B.Heinzelman, A.P.Chandrakasan, (2000), "Energy-efficient communication protocols for wireless micro sensor networks", in Proceeding of the 33rd Hawaii International Conference on System Sciences, Pages 1-10.

12) Goofing Hour, K.Wendy Tang, (2006), "Evaluation of LEACH protocol subject to different traffic models", in 1st International Conference on Next Generation Network.

13) O.Younis, S.Fahmy, (2004), "HEED: A hybrid energy-efficient distributed clustering approach for adhoc sensor networks", IEEE Transactions on Mobile Computing, Volume 3, Number 4, Pages 366-379. 00.511 .52100200300400500600 700800900 Effective Lifetime x 103 Coverage Area x 103 Network Lifetime HEED 100 Nodes HEED 200 Nodes CPHRP 100 Nodes CPHRP 200 Nodes 02004006008001000100200300 $\begin{array}{lllllll}400 & 500 & 600 & 700 & 800 & 900 & \text { Effective Rounds }\end{array}$ Coverage Rate x 103 Effective Number of Rounds
HEED CPHRP Copyright to IJIREEICE www.ijireeice.com 406

14) C.F.Li, G.H.Chen, (2007), "An uneven clusterbased routing protocol for wireless sensor networks", Chinese Journal of Computers, Volume 30, Number 1, Pages 27-36.

15) J.Ibriq, I.Mahgoub, (2004), "Cluster-based routing in wireless sensor networks: issues and challenges", in Proceedings of the Symposium on Performance Evaluation of Computer Telecommunication Systems, Pages 759-766.

16) P.Tillapart, S.Thammarojsakul, (2005), "An approach to hybrid clustering and routing in wireless sensor networks," in Proceedings of the IEEEAC, Pages 1-8.

17) R.S.Chang, C.J.Kuo, (2006), "An energy efficient routing mechanism for wireless sensor networks", in Proceedings of the 20th International Conference on Advanced Information Networking and Applications, Pages 308-312.

18) Osama Youngish, Sonia Filmy, "Distributed clustering in adhoc sensor networks: a hybrid energy-efficient approach", Purdue University, USA.

19) N.Jamal, Al-Karakas Ahmed, E.Kamal, "Routing techniques in wireless sensor networks: a survey", Iowa State University.

20) H.Kour, A.K.Sharma, (2010), "Hybrid energy efficient distributed protocol for heterogeneous wireless sensor network", International Journal of Computer Applications, Volume 4, Number 6, Pages 0975 - 8887 .

21) Jamal Iberia, lead Mahfouz, (2004), "Cluster based routing in wireless sensor networks: issues and challenges", SPECTS ${ }^{\text {ee }}$ 04, Pages 759-766.

22) Osama Youngish, Sonia Filmy, (2004), "HEED: A hybrid energy efficient distributed clustering approach for adhoc networks", IEEE Transactions on Mobile Computing, Volume 3, Number 4, Pages 366-369.

23) Caching, Howe, Willie, M.Gerla, (1997), "Routing in clustered multichip mobile wireless networks with fading channel", in Proceeding of IEEE Singapore International Conference on Networks (SICON ,07), Pages 197- 211.

24) V.Rodoplu, T.H.Meng, (1999), "Minimum energy mobile wireless networks", IEEE Journal on 
Selected Areas in Communications, Volume 17, Number 8, Pages 33-44.

25) R.Wattenhofer, Lela, P.Bahl, Y.M.Wang, (2001), "Distributed topology control for power efficient operation in multichip adhoc networks", IEEE Magazine, Volume 3.

26) S.R.Gandham, M.Dawande, R.Prakash, S.Venkatesan, (2003), "Energy efficient schemes for wireless sensor networks with multiple mobile base stations", in Proceedings of IEEE Global Telecommunications Conference, Pages 377-381.

27) D.Ganesan, R.Govindan, S.Shenker, Diestrum, (2001), "Wireless sensor networks", ACM Mobile Computing and Communications Review, Volume 5, Number 4.

28) S.K.Singh, M.P.Singh, D.K.Singh, (2010), "A survey of energy efficient hierarchical cluster based routing in wireless sensor networks", International Journal of Advanced Networking and Application, Volume 2, Issue 2, Pages 570 580.

29) Ming Liu, Japanning Cao, Guitar Chen, Xiaoping Wang, (2009), “An energy aware routing protocol in wireless sensor networks", Sensors 2009, Volume 9, Pages 445-462.
30) Jamal Karaka, A.E.Kamal, (2004), "Routing techniques in wireless sensor networks: a survey", IEEE Communications Magazine, Volume 11, Number 6, Pages 6-28.

31) Osama You is, Sonia Filmy, (2004), "HEED: A hybrid, energy efficient distributed clustering approach for adhoc networks", IEEE Transactions on Mobile Computing, Volume 3, Number 4, Pages 366-369.

32) K.Akkaya, M.Younis, (2003), “An energy-aware QoS routing protocol for wireless sensor networks", in Proceedings of the IEEE Workshop on Mobile and Wireless Networks (MWN 2003).

33) Qulin, J.Aslam, Drugs, (2001), "Hierarchical power aware routing in sensor networks", in Proceedings of the DIMACS Workshop.

34) Niangua Lin, Munching Liao, (2010), “A clustering patch hierarchical routing protocol for wireless sensor networks" in the 5th International Conference on Computer Science and Education.

35) Dewey, S.Kaplan, H.Chan, (2008), "Energy efficient clustering algorithms for wireless sensor networks", in Proceedings of IEEE Communications Society (ICC 2008), Pages 236240. 